\title{
Active Surveillance of Adverse Events in Healthcare Workers Recipients After Vaccination with COVID-19 BNT162b2 Vaccine (Pfizer-BioNTech, Comirnaty): A Cross-Sectional Study
}

\author{
Giancarlo Ripabelli ${ }^{1,3}$ - Manuela Tamburro ${ }^{1} \cdot$ Nicandro Buccieri $^{2} \cdot$ Carmen Adesso $^{3} \cdot$ Valeria Caggiano $^{3}$. \\ Fabio Cannizzaro ${ }^{3}$. Michela Anna Di Palma ${ }^{3}$. Gloria Mantuano ${ }^{3}$. Valeria Giovanna Montemitro ${ }^{3} \cdot$ Anna Natale $^{3}$. \\ Leonardo Rodio ${ }^{3} \cdot$ Michela Lucia Sammarco $^{1}$
}

Accepted: 1 October 2021 / Published online: 9 October 2021

(c) The Author(s), under exclusive licence to Springer Science+Business Media, LLC, part of Springer Nature 2021

\begin{abstract}
In this cross-sectional study, adverse events after the first and second dose of BNT162b2 mRNA (Pfizer-BioNTech, Comirnaty) vaccine against coronavirus disease 2019 were investigated among employees of clinics in central Italy. A 42 -items questionnaire was administrated to vaccine recipients. Adverse events were classified based on severity and occurrence as reported in the literature. A descriptive/univariate analysis using Chi-square or Fisher's Exact tests was performed. Odds ratio (OR) and $95 \%$ confidence intervals were calculated to assess risk factors. 340 individuals $(61.5 \%$ females; median age 49 years) participated. Adverse events were reported by $279(82 \%)$ and $281(82.6 \%)$ individuals as induced by the first and second dose, respectively. Mild reactions were mainly reported $(80.9 \%$ and $80.3 \%)$, followed by moderate $(11.8 \%$ and $37.1 \%)$ and severe (3.8\% and $4.7 \%)$. Adverse events were identical to those already described as very common ( $81.8 \%$ and $80.6 \%$ ), although vaccine-coincidental events not cited in the literature were reported by $6 \%$ and $15.6 \%$ following each dose. Age $\leq 55$ years was a risk factor for any adverse event after each injection (ORs: 2.942 and 2.818), as well as female sex for those mild (ORs: 1.856 and 2.818) and common (ORs: 3.452 and 2.145). Findings were consistent with national reports as most of the adverse events were mild and associated with female sex and young age, while investigations are needed for reactions not described elsewhere. Data are useful to support the vaccine safety profile, also because largely targeted healthcare personnel more skilled than general population in self-diagnosis of health-related issues.
\end{abstract}

Keywords BNT162b2 mRNA vaccine $\cdot$ First dose $\cdot$ Sex differences $\cdot$ Safety $\cdot$ Second dose $\cdot$ Younger adults

Giancarlo Ripabelli

ripab@unimol.it

Manuela Tamburro

manuela.tamburro@unimol.it

Nicandro Buccieri

nicandrobuccieri@gmail.com

Carmen Adesso

carmen.adesso@hotmail.it

Valeria Caggiano

valeria.caggiano93@gmail.com

Fabio Cannizzaro

fabio.cannizzaro84@libero.it

Michela Anna Di Palma

dipalmamichela@hotmail.it

Gloria Mantuano

gloria.mantuano@hotmail.it
Valeria Giovanna Montemitro

valeriamontemitro1@gmail.com

Anna Natale

anna-natale68@virgilio.it

Leonardo Rodio

leonardo.rodio.rh@gmail.com

Michela Lucia Sammarco

sammarco@unimol.it

1 Department of Medicine and Health Sciences "Vincenzo Tiberio", University of Molise, Via De Sanctis, 86100 Campobasso, Italy

2 Casa Di Cura Istituto Europeo Di Riabilitazione, Isernia, Italy

3 School of Specialization in Hygiene and Preventive Medicine, University of Molise, Campobasso, Italy 


\section{Introduction}

Coronavirus 2019 (COVID-19) disease, at time of writing (August 6, 2021) globally affected more than 202 million of people and caused more than 4 million deaths [1], while about 4 million confirmed cases and 128,220 deaths occurred in Italy. Beyond the public health measures implemented for containing the pandemic [2], huge efforts have been undertaken for the vaccine development and administration $[3,4]$, being the most effective way to prevent disease and outcomes [5].

Data updated to August 3, 2021, reported that 21 vaccines were approved by at least in one world country, of which eight were inactivated vaccines, six non-replicating viral vector, four protein subunits, and three RNA-based vaccines [6]. On December 11, 2020, the Food and Drug Administration (FDA) issued an Emergency Use Authorization for BNT162b2 (Pfizer-BioNTech, Comirnaty) mRNA COVID-19 vaccine [7], and on December 21, 2020, the European Medicines Agency (EMA) authorised the vaccine to people from 16 years of age [8]. On December 22, the Italian Medicines Agency also authorised the marketing of Comirnaty vaccine [9], and the vaccination campaign started on December 27, 2020. As of August 6, 2021, in Italy, 70,587,195 doses have been administered [10].

Despite being safe, sustainable, and effective, vaccines could be accompanied with risks of coincidental adverse events, which may not necessarily have a causal relationship with their use [11]. For candidate vaccines, safety assessment is integrated into all stages of the clinical development pipeline, and once they are deployed into the population [12]. The interim phase $1 / 2$ safety results revealed that mRNA-based vaccines generally led to pain at the injection site as a very common feature, followed by headache, myalgia, fatigue, and fever [13, 14]. During mass vaccination campaign, novel findings on the safety profiles of each vaccine are being accumulated, including that people experienced more adverse events after the second dose of Comirnaty vaccine than the first [15]. In Italy, adverse reactions were voluntarily reported by vaccine recipients to the pharmacovigilance surveillance on COVID-19 vaccines established by the Italian Medicines Agency [16], and reports were published monthly for each vaccine. Conversely, research studies on vaccine-related adverse events are limited yet [17], and mostly retrieved with an electronic search in the public Italian Medicines Agency database [18].

In this study, an active surveillance was conducted among employees of clinics in central Italy to evaluate occurrence of any adverse event after both injections of Comirnaty vaccine, and to investigate reactions previously unrecognised in the literature or in the summary of product characteristics.
The findings may provide new insights on the vaccine safety profile, enabling risk data stratification by sex, age, professional activity, comorbidities, and pharmacological treatments of the participants.

\section{Materials and Methods}

\section{Study Design and Setting}

This was a retrospective study based on data anonymously collected during February-March 2021 by enrolling employees at five private health clinics, three in Molise and two in Abruzzo regions, central Italy. The research was designed to investigate on each adverse event after immunization with Comirnaty vaccine administered between January and March 2021. The inclusion criteria for the participation were (i) to sign an informed consent agreeing to the study aims and provide anonymous data on vaccination; (ii) to be employed in a clinic identified, and (iii) to have received and completed the Comirnaty vaccination.

\section{Questionnaire Methodology}

Participants were asked to reply to questions formulated by trained interviewers via the telephone. A novel questionnaire was developed, which was tested before administration in a pilot study among healthcare personnel for its validation (data not reported). The questionnaire comprised 42 questions, structured into different sections as follows: sociodemographic characteristics; COVID-19 history; individual's vaccination history in the previous 6 months; follow-up after the first and second injection of Comirnaty vaccine; reporting of adverse event following COVID vaccination; acute or chronic pre-existing diseases and related treatment; and medical surveillance related to results of swab testing during or after COVID vaccination.

\section{Questions on Adverse Events After the First and Second Vaccine Dose}

Participants provided date of the dose administration, and information on any adverse event listed in the questionnaire and classified into mild, moderate, and severe, according to the trial design conducted by Pfizer [19] and elsewhere [20]. Mild events included pain/redness/swelling at the injection site, fatigue, headache, fever $\leq 38^{\circ} \mathrm{C}$, chills, abdominal pain, nausea, insomnia, and sleep disorders. Moderate events were fever $>38^{\circ} \mathrm{C}$ and $<39{ }^{\circ} \mathrm{C}$, lymphadenopathy, myalgia/arthralgia, localized urticaria, vomiting, and diarrhoea. Severe events comprised generalized urticaria, angioedema, oedema glottidis, dyspnoea, anaphylaxis, neuropathy, paraesthesia, and fever $\geq 39^{\circ} \mathrm{C}$. 
The adverse events were also grouped into very common $(\geq 1 / 10)$, common $(\geq 1 / 100$ to $<1 / 10)$, uncommon $(\geq 1 / 1000$ to $<1 / 100)$, and rare $(\geq 1 / 10,000$ to $<1 / 1000)$, according to the European Medicines Agency [21]. Any additional adverse event not listed and spontaneously reported by the participants was annotated for both doses. After each injection, participants were asked to indicate onset and resolution of each adverse event. There were also questions on medication used for the treatment of any symptom/sign after vaccine injections, as well as those used prior to vaccine administration.

\section{Statistics}

A descriptive analysis was performed, calculating absolute/ relative (\%) frequencies, and mean, median and range for qualitative and quantitative variables, respectively. Chisquare and Fisher's Exact tests were used to evaluate the association between adverse events and any factor. Chisquare test was also applied to evaluate differences between occurrence of adverse events after each injection. The analyses were carried out using IBM SPSS Statistics Software, version 26.0, and considered significant for $\mathrm{p}<0.05$. Multivariate logistic regression (with stepwise method with backward elimination) enabled to assess the association between adverse events and any variable, calculating Odds Ratios (ORs) and 95\% confidence intervals (95\% CI).

\section{Results}

\section{Study Sample}

404 individuals were identified as had undergone Comirnaty vaccination, although $64(15.8 \%)$ subjects were excluded because did not fit with the inclusion criteria. The 340 remaining subjects participated to the study (response rate $84.1 \%)$. The enrolled subjects were aged $49.2 \pm 13.4$ years (median 49 years; range 20-79 years), 209 (61.5\%) were females, and $263(77.3 \%)$ were healthcare workers (HCWs). $25 \%(\mathrm{n}=86)$ of the vaccine recipients belonged to the age group of 40-49 years, followed by $22 \%$ at age 60-69 years $(\mathrm{n}=76)$ and $50-59$ years $(\mathrm{n}=75)$.

$147(43.2 \%)$ participants declared suffering from comorbidities, and the most frequent were hypertension $(n=55$, $37.4 \%)$, allergies $(n=42,28.6 \%)$, thyroid disorders $(n=34$, $23.1 \%)$, hypercholesterolaemia $(\mathrm{n}=15,10.2 \%)$, and diabetes $(n=9,6.1 \%)$. At the time of the interview, $42.6 \%(n=145)$ of participants who had received their first dose was under treatment for acute/chronic diseases, with thyroid $(n=25$,
$17.2 \%)$ and antihypertensive $(\mathrm{n}=20,13.8 \%)$ medication as the most prevalent treatment.

In the 6 months prior to the first Comirnaty dose, $89(26.2 \%)$ individuals had received other vaccines: 77 $(86.5 \%)$ anti-influenza, $11(12.3 \%)$ anti-influenza and anti-pneumococcal vaccines, and one (1.1\%) anti-HBV. Before the first injection, 18 (5.3\%) workers had a COVID19 diagnosis confirmed by molecular PCR-based testing between March and November 2020, and 15 (83.3\%) had developed COVID-19 symptoms, although none had severe or critical disease or required hospitalization. Twenty percent $(n=68)$ of participants were subjected to a nasopharyngeal swab for SARS-CoV-2 testing for a screening program or in another context, with $57(n=83.8 \%)$ and $11(16.2 \%)$ who undergone a rapid antigenic test and molecular swab test, respectively. Results were negative in all cases. Despite the vaccination, seven $(2.1 \%)$ individuals reported COVID-related symptoms (unknown time of onset), including dry cough, fever $>38^{\circ} \mathrm{C}$, myalgia, and gastrointestinal disorders; of these, three subjects had a previous confirmed diagnosis of COVID-19. All except seven participants $(n=333,97.9 \%)$ reported their willingness to undergo a serological test from venous sampling. Notably, at the time of the interview, 84 (24.7\%) already undergone a serological testing after the second dose (complete cycle) for the evaluation of the anti-spike IgG seroconversion.

\section{Vaccine-Related Adverse Events After the First Dose}

279 (82.1\%; mean age $47.6 \pm 13.1$ years; range 20-79 years) experienced at least one adverse event after the first dose, more frequently in females $(n=181,64.9 \%)$. $64 \%(n=179)$ reported a single adverse event, followed by two $(n=45,16.2 \%)$, and three $(n=28,10 \%)$ events. Vaccine recipients aged $40-49$ years $(n=72,25.8 \%)$ more often reported adverse events, particularly $153(54.8 \%)$ people aged $\leq 49$ years, and $196(70.3 \%)$ of recipients aged $\leq 55$ and $>55$ years, respectively.

After the first dose, at least one adverse event was most frequently reported by nurses $(n=74,26.5 \%)$, followed by medical doctors $(n=58,20.8 \%)$, social health operators $(n=41,14.7 \%)$, administrators $(n=32,11.5 \%)$, and physiotherapists $(n=23,8.2 \%)$.

Mild adverse events were reported by 275 (98.6\%) individuals, followed by moderate $(\mathrm{n}=40,14.3 \%)$ and severe $(\mathrm{n}=13,4.7 \%)$. Pain, redness and swelling at the injection site $(n=265,77.9 \%)$ were mostly reported among mild events, followed by fatigue, headache, and chills (Table 1). Moderate events mainly included myalgia/arthralgia $(n=31,9.1 \%)$, followed by lymphadenopathy, diarrhoea and local urticaria while paraesthesia and generalized 
Table 1 Vaccine-related adverse events following the first and the second dose of Comirnaty vaccine

\begin{tabular}{|c|c|c|c|}
\hline Vaccine-related adverse events & $\begin{array}{l}\text { I dose } \\
\mathrm{N}(\%)\end{array}$ & $\begin{array}{l}\text { II dose } \\
\mathrm{N}(\%)\end{array}$ & P-value* \\
\hline Mild & $275(80.9)$ & $273(80.3)$ & 0.845 \\
\hline $\begin{array}{l}\text { Pain, redness and swelling at } \\
\text { the injection site }\end{array}$ & $265(77.9)$ & $223(65.6)$ & $<0.001$ \\
\hline Fatigue & $66(19.4)$ & $135(39.7)$ & $<0.001$ \\
\hline Headache & $52(15.3)$ & $98(28.8)$ & $<0.001$ \\
\hline Fever $\leq 38{ }^{\circ} \mathrm{C}$ & $5(1.5)$ & $46(13.5)$ & $<0.001$ \\
\hline Chills & $20(5.9)$ & $90(26.5)$ & $<0.001$ \\
\hline Abdominal pain & $3(0.9)$ & $26(7.6)$ & $<0.001$ \\
\hline Nausea & $7(2.1)$ & $33(9.7)$ & $<0.001$ \\
\hline Insomnia & $5(1.5)$ & $8(2.4)$ & 0.401 \\
\hline Sleep disorders & $8(2.4)$ & $20(5.9)$ & $\mathbf{0 . 0 2 0}$ \\
\hline Moderate & $40(11.8)$ & $126(37.1)$ & $<0.001$ \\
\hline Fever $>38^{\circ} \mathrm{C}$ and $<39^{\circ} \mathrm{C}$ & $0(0)$ & $23(6.7)$ & $<0.001$ \\
\hline Lymphadenopathy & $8(2.4)$ & $23(6.7)$ & $<0.001$ \\
\hline Myalgia/Arthralgia & $31(9.1)$ & $115(33.8)$ & $<0.001$ \\
\hline Local urticaria & $2(0.6)$ & $2(0.6)$ & $\begin{array}{l}\text { Not com } \\
\text { putable }\end{array}$ \\
\hline Vomit & $0(0)$ & $3(0.8)$ & 0.082 \\
\hline Diarrhoea & $7(2.1)$ & $21(6.2)$ & $\mathbf{0 . 0 0 7}$ \\
\hline Severe & $13(3.8)$ & $16(4.7)$ & 0.569 \\
\hline Dyspnoea & $0(0)$ & $1(0.3)$ & 0.317 \\
\hline Paraesthesia & $11(3.2)$ & $11(3.2)$ & $\begin{array}{l}\text { Not com- } \\
\text { putable }\end{array}$ \\
\hline Generalized urticaria & $3(0.9)$ & $0(0)$ & 0.082 \\
\hline Fever $\geq 39{ }^{\circ} \mathrm{C}$ & $0(0)$ & $4(1.2)$ & 0.045 \\
\hline
\end{tabular}

*Chi-square test; significant results $(\mathrm{p}<0.05)$ are indicated in bold

urticaria were indicated among severe events (Table 1). Mild and moderate events generally occurred after 4-12 h from the first injection (Table 2a) and resolved after 12 and 12-24 h from the occurrence (Table $2 b$ ).

After the first dose, participants largely reported common events $(\mathrm{n}=278,81.8 \%)$, whilst common and uncommon events accounted for $12.6 \%(n=43)$ and $2.4 \%(n=8)$, respectively.

$26(7.6 \%)$ participants reported additional temporarily coincidental adverse events and not previously described in the literature, including cognitive or brain fog $(n=4)$, face flushing and dizziness (each $\mathrm{n}=2$ ). Hot flashes, dysgeusia, dysosmia, breathlessness, asthenia from exertion, ipsilateral upper limb bursitis, loss of vision or amaurosis fugax, intense pain in ipsilateral upper limb, erectile dysfunction, lack of appetite, irritability, general malaise, hypertensive peak, precordial pain, generalized itching, vasovagal syncope, dry eye, and arm swelling were also reported each by one recipient.

\section{Adverse Events Reporting, Premedication, and Medication at the First Dose}

$37(13.3 \%)$ out of 279 individuals reported adverse events to medical services, specifically to occupational physicians $(n=34,91.9 \%)$, family doctors $(n=2,5.4 \%)$, or emergency $(\mathrm{n}=1,2.7 \%)$.

$27(7.9 \%)$ participants received a medication prior to the first dose, with cortisone $(n=19,70.4 \%)$, antihistamines $(n=7,25.9 \%)$, or antihypertensives $(n=1,3.7 \%)$. Furthermore, $60(21.5 \%)$ participants reporting adverse events undergone a treatment for these reactions, most commonly using non-steroidal anti-inflammatory drugs (NSAIDs, $\mathrm{n}=26,43.3 \%)$ and paracetamol $(\mathrm{n}=20,33.3 \%)$.

\section{Vaccine-Related Adverse Events After the Second Dose}

Adverse events after the second injection were reported by $281(82.6 \%)$ individuals aged $47.8 \pm 13.1$ years (range 20-74 years), and most frequently by females $(n=181$, $64.6 \%$ ). A single adverse event was most commonly reported by $31.3 \%(\mathrm{n}=88)$ of the participants, followed by two $(n=50,17.8 \%)$, and three $(n=41,14.6 \%)$ events.

Vaccine recipients aged $40-49$ years more often $(n=71$, $25.3 \%$ ) reported adverse events. Particularly, incidents were referred by $150(54.1 \%)$ people aged $\leq 49$ years, and $70.1 \%$ $(n=197)$ of those aged $\leq 55$ years. Participants reporting adverse events after the second dose were most frequently nurses $(n=73,25.9 \%)$, followed by medical doctors $(n=64$, $22.8 \%)$, social health operators $(n=42,14.9 \%)$, and administrators $(n=31,11.0 \%)$.

At the time of the interview, $42.0 \%(n=143)$ of participants who received the second dose were under treatment for an acute or chronic disease, with antihypertensive $(n=23$, $16.1 \%)$ and thyroid medications $(\mathrm{n}=22,15.4 \%)$ as the most prevalent treatments.

Adverse events after the second dose $(\mathrm{n}=281)$ were most commonly mild, being reported by $273(97.2 \%)$, followed by moderate $(n=126,44.8 \%)$ and severe $(n=16,5.7 \%)$. Pain, redness and swelling at the injection site were the most common mild reactions $(n=222,65.3 \%)$, followed by fatigue and headache (Table 1). Among moderate adverse events, 115 (33.8\%) subjects reported myalgia/arthralgia and $23(6.8 \%)$ lymphadenopathy, while paraesthesia and fever $>39^{\circ} \mathrm{C}$ were reported among the severe events (Table 1).

Following the second injection, mild and moderate events most frequently occurred after 4-12 and 12-24 h (Table 3a). $72 \%$ of adverse events lasted after 12-24 h, and only lymphadenopathy and abdominal pain resolved after $48-72 \mathrm{~h}$ and above $72 \mathrm{~h}$, respectively (Table $3 \mathrm{~b}$ ). 
Table 2 Onset (a) and resolution (b) of adverse events following the first vaccine injection

\begin{tabular}{|c|c|c|c|c|c|c|c|}
\hline (a) Onset & $\begin{array}{l}\leq 1 \mathrm{~h} \\
\mathrm{~N}(\%)\end{array}$ & $\begin{array}{l}1-4 \mathrm{~h} \\
\mathrm{~N}(\%)\end{array}$ & $\begin{array}{l}4-12 \mathrm{~h} \\
\mathrm{~N}(\%)\end{array}$ & $\begin{array}{l}12-24 \mathrm{~h} \\
\mathrm{~N}(\%)\end{array}$ & $\begin{array}{l}24-48 \mathrm{~h} \\
\mathrm{~N}(\%)\end{array}$ & $\begin{array}{l}>48 \mathrm{~h} \\
\mathrm{~N}(\%)\end{array}$ & $\begin{array}{l}\text { Missing data } \\
\mathrm{N}(\%)\end{array}$ \\
\hline \multicolumn{8}{|l|}{ Mild } \\
\hline $\begin{array}{l}\text { Pain, redness and swelling at the } \\
\text { injection site }(\mathrm{n}=265)\end{array}$ & $30(11.3)$ & $68(25.7)$ & $109(41.1)$ & $50(18.9)$ & $7(2.6)$ & & $1(0.4)$ \\
\hline Fatigue $(n=66)$ & $3(4.5)$ & $12(18.2)$ & $20(30.3)$ & $17(25.8)$ & $7(10.6)$ & $3(4.5)$ & $4(6.1)$ \\
\hline Headache $(\mathrm{n}=52)$ & $10(19.2)$ & $10(19.2)$ & $13(25.0)$ & $12(23.1)$ & $5(9.7)$ & $1(1.9)$ & $1(1.9)$ \\
\hline Fever $\leq 38^{\circ} \mathrm{C}(\mathrm{n}=5)$ & & & $2(40.0)$ & $3(60.0)$ & & & \\
\hline Chills $(n=20)$ & $1(5.0)$ & $3(15.0)$ & $4(20.0)$ & $11(55.0)$ & $1(5.0)$ & & \\
\hline Abdominal pain $(\mathrm{n}=3)$ & $1(33.3)$ & & $1(33.3)$ & & $1(33.3)$ & & \\
\hline Nausea $(n=7)$ & $1(14.3)$ & $3(42.8)$ & $2(28.6)$ & $1(14.3)$ & & & \\
\hline Insomnia $(\mathrm{n}=5)$ & & & $3(60.0)$ & $2(40.0)$ & & & \\
\hline Sleep disorders $(n=8)$ & & & $6(75.0)$ & & $1(12.5)$ & $1(12.5)$ & \\
\hline \multicolumn{8}{|l|}{ Moderate } \\
\hline Lymphadenopathy $(\mathrm{n}=8)$ & & $1(12.5)$ & $2(25.0)$ & $1(12.5)$ & $1(12.5)$ & $2(25.0)$ & $1(12.5)$ \\
\hline Myalgia/Arthralgia $(n=31)$ & $2(6.4)$ & $1(3.2)$ & $7(22.6)$ & $11(35.6)$ & $3(9.7)$ & $6(19.3)$ & $1(3.2)$ \\
\hline Local urticaria $(\mathrm{n}=2)$ & $2(100)$ & & & & & & \\
\hline Diarrhoea $(n=7)$ & $1(14.3)$ & & $1(14.3)$ & $1(14.3)$ & $1(14.3)$ & $3(42.8)$ & \\
\hline \multicolumn{8}{|l|}{ Severe } \\
\hline Paraesthesia $(n=11)$ & 8 (72.7) & & & $1(9.1)$ & $2(18.2)$ & & \\
\hline Generalized urticaria $(n=3)$ & $3(\mathbf{1 0 0})$ & & & & & & \\
\hline (b) Resolution & $\begin{array}{l}<12 \mathrm{~h} \\
\mathrm{~N}(\%)\end{array}$ & $\begin{array}{l}12-24 \mathrm{~h} \\
\mathrm{~N}(\%)\end{array}$ & $\begin{array}{l}24-48 \mathrm{~h} \\
\mathrm{~N}(\%)\end{array}$ & & $\begin{array}{l}48-72 \mathrm{~h} \\
\mathrm{~N}(\%)\end{array}$ & $\begin{array}{l}>72 \mathrm{~h} \\
\mathrm{~N}(\%)\end{array}$ & $\begin{array}{l}\text { Missing data } \\
\mathrm{N}(\%)\end{array}$ \\
\hline \multicolumn{8}{|l|}{ Mild } \\
\hline $\begin{array}{l}\text { Pain, redness and swelling at the } \\
\text { injection site }(n=265)\end{array}$ & $44(16.6)$ & $85(32.1)$ & $82(30.9)$ & & $38(14.3)$ & $15(5.7)$ & $1(0.4)$ \\
\hline Fatigue $(n=66)$ & $7(10.6)$ & $25(37.9)$ & $14(21.2)$ & & $7(10.6)$ & $9(13.6)$ & $4(6.1)$ \\
\hline Headache $(n=52)$ & $19(36.5)$ & $14(26.9)$ & $8(15.4)$ & & $5(9.6)$ & $6(11.6)$ & \\
\hline Fever $\leq 38^{\circ} \mathrm{C}(\mathrm{n}=5)$ & $2(40.0)$ & $2(40.0)$ & $1(20.0)$ & & & & \\
\hline Chills $(n=20)$ & $8(40.0)$ & $3(15.0)$ & $4(20.0)$ & & $3(15.0)$ & $2(10.0)$ & \\
\hline Abdominal pain $(n=3)$ & $1(33.3)$ & & & & $2(66.7)$ & & \\
\hline Nausea $(n=7)$ & $2(28.6)$ & $1(14.2)$ & $2(28.6)$ & & & $2(28.6)$ & \\
\hline Insomnia $(\mathrm{n}=5)$ & $4(80.0)$ & $1(20.0)$ & & & & & \\
\hline Sleep disorders $(\mathrm{n}=8)$ & $6(75.0)$ & $1(12.5)$ & & & & $1(12.5)$ & \\
\hline \multicolumn{8}{|l|}{ Moderate } \\
\hline Lymphadenopathy $(\mathrm{n}=8)$ & & $1(12.5)$ & $1(12.5)$ & & $2(25.0)$ & $3(37.5)$ & $1(12.5)$ \\
\hline Myalgia/Arthralgia $(\mathrm{n}=31)$ & $2(6.4)$ & $10(32.2)$ & $7(22.6)$ & & $4(12.9)$ & 7 (22.6) & $1(3.3)$ \\
\hline Local urticaria $(n=2)$ & $2(100)$ & & & & & & \\
\hline Diarrhoea $(\mathrm{n}=7)$ & $1(14.2)$ & $2(28.6)$ & & & $2(28.6)$ & $2(28.6)$ & \\
\hline \multicolumn{8}{|l|}{ Severe } \\
\hline Paraesthesia $(n=11)$ & $8(72.7)$ & $1(9.1)$ & & & & $1(9.1)$ & $1(9.1)$ \\
\hline Generalized urticaria $(n=3)$ & $1(33.3)$ & & $1(33.3)$ & & & & $1(33.4)$ \\
\hline
\end{tabular}

The higher frequencies among the listed options are reported in bold

Differences in the occurrence of adverse events after the first and second dose were observed (Table 1), particularly occurrence of fever $>38^{\circ} \mathrm{C}$ and $<39{ }^{\circ} \mathrm{C}$, lymphadenopathy, myalgia/arthralgia, and diarrhoea significantly increased after the second dose. Among the serious events, fever $>39{ }^{\circ} \mathrm{C}$ was more frequently reported after the second injection as compared to the first. Furthermore, while mild events increased in their occurrences after the second dose, the reporting of pain, redness and swelling at the injection site decreased at the second dose (Table 1). Of note, fever $>38{ }^{\circ} \mathrm{C}$ and $<39{ }^{\circ} \mathrm{C}$, vomit, dyspnoea, and 
Table 3 Onset (a) and resolution (b) of adverse events following the second vaccine injection

\begin{tabular}{|c|c|c|c|c|c|c|c|}
\hline (a) Onset & $\begin{array}{l}\leq 1 \mathrm{~h} \\
\mathrm{~N}(\%)\end{array}$ & $\begin{array}{l}1-4 \mathrm{~h} \\
\mathrm{~N}(\%)\end{array}$ & $\begin{array}{l}4-12 \mathrm{~h} \\
\mathrm{~N}(\%)\end{array}$ & $\begin{array}{l}12-24 \mathrm{~h} \\
\mathrm{~N}(\%)\end{array}$ & $\begin{array}{l}24-48 \mathrm{~h} \\
\mathrm{~N}(\%)\end{array}$ & $\begin{array}{l}>48 \mathrm{~h} \\
\mathrm{~N}(\%)\end{array}$ & $\begin{array}{l}\text { Missing data } \\
\mathrm{N}(\%)\end{array}$ \\
\hline \multicolumn{8}{|l|}{ Mild } \\
\hline $\begin{array}{l}\text { Pain, redness and swelling at the } \\
\text { injection site }(n=223)\end{array}$ & $37(16.6)$ & $63(28.2)$ & $87(39.1)$ & $31(13.9)$ & $4(1.8)$ & & $1(0.4)$ \\
\hline Fatigue $(\mathrm{n}=135)$ & $4(2.9)$ & $19(14.1)$ & $42(31.1)$ & $49(36.3)$ & $15(11.1)$ & $6(4.5)$ & \\
\hline Headache $(n=98)$ & $3(3.1)$ & $16(16.3)$ & 34 (34.7) & $31(31.6)$ & $9(9.2)$ & $5(5.1)$ & \\
\hline Fever $\geq 38{ }^{\circ} \mathrm{C}(\mathrm{n}=46)$ & & $2(4.3)$ & $19(41.3)$ & $18(39.1)$ & $5(10.9)$ & $1(2.2)$ & $1(2.2)$ \\
\hline Chills $(\mathrm{n}=90)$ & $3(3.3)$ & $9(10.0)$ & $37(41.1)$ & 33 (36.7) & $6(6.7)$ & & $2(2.2)$ \\
\hline Abdominal pain $(n=26)$ & $2(7.7)$ & $3(11.5)$ & $2(7.7)$ & $10(38.5)$ & $5(19.2)$ & $2(7.7)$ & $2(7.7)$ \\
\hline Nausea $(\mathrm{n}=33)$ & $1(3.0)$ & $3(9.1)$ & $8(24.2)$ & $13(39.4)$ & $4(12.1)$ & $2(6.1)$ & $2(6.1)$ \\
\hline Insomnia $(\mathrm{n}=8)$ & & $1(12.5)$ & 7 (87.5) & & & & \\
\hline Sleep disorders $(n=20)$ & & $2(10.0)$ & $10(50.0)$ & $6(30.0)$ & $2(10.0)$ & & \\
\hline \multicolumn{8}{|l|}{ Moderate } \\
\hline Fever $>38^{\circ} \mathrm{C}$ and $<39^{\circ} \mathrm{C}(\mathrm{n}=23)$ & & $1(4.3)$ & $10(43.5)$ & $10(43.5)$ & $2(8.7)$ & & \\
\hline Lymphadenopathy $(\mathrm{n}=23)$ & $1(4.3)$ & & $1(4.3)$ & $12(52.2)$ & $4(17.4)$ & $3(13.1)$ & $2(8.7)$ \\
\hline Myalgia/Arthralgia $(n=115)$ & & $10(8.7)$ & $38(33.0)$ & $50(43.5)$ & $15(13.0)$ & $1(0.9)$ & $1(0.9)$ \\
\hline Local urticaria $(n=2)$ & $1(50.0)$ & & $1(50.0)$ & & & & \\
\hline Vomit $(n=3)$ & & & & $3(100)$ & & & \\
\hline Diarrhoea $(n=21)$ & & $2(9.5)$ & $1(4.8)$ & $10(47.7)$ & $4(19.0)$ & $4(19.0)$ & \\
\hline \multicolumn{8}{|l|}{ Severe } \\
\hline Dyspnoea $(\mathrm{n}=1)$ & & & & & & $1(100)$ & \\
\hline Paraesthesia $(n=11)$ & $3(27.3)$ & $1(9.1)$ & $3(27.3)$ & $2(18.1)$ & $1(9.1)$ & $1(9.1)$ & \\
\hline Fever $\geq 39^{\circ} \mathrm{C}(\mathrm{n}=4)$ & & & $2(50.0)$ & $2(50.0)$ & & & \\
\hline b) Resolution & $\begin{array}{l}<12 \mathrm{~h} \\
\mathrm{~N}(\%)\end{array}$ & $\begin{array}{l}12-24 \mathrm{~h} \\
\mathrm{~N}(\%)\end{array}$ & $\begin{array}{l}24-48 \text { h } \\
\mathrm{N}(\%)\end{array}$ & & $\begin{array}{l}48-72 \mathrm{~h} \\
\mathrm{~N}(\%)\end{array}$ & $\begin{array}{l}>72 \mathrm{~h} \\
\mathrm{~N}(\%)\end{array}$ & $\begin{array}{l}\text { Missing data } \\
\mathrm{N}(\%)\end{array}$ \\
\hline \multicolumn{8}{|l|}{ Mild } \\
\hline $\begin{array}{l}\text { Pain, redness and swelling at the injec- } \\
\text { tion site }(n=223)\end{array}$ & $42(18.8)$ & $68(30.5)$ & $57(25.6)$ & & $31(13.9)$ & $24(10.8)$ & $1(0.4)$ \\
\hline Fatigue $(\mathrm{n}=135)$ & $13(9.6)$ & $42(31.1)$ & $26(19.3)$ & & $20(14.8)$ & $33(24.5)$ & $1(0.7)$ \\
\hline Headache $(\mathrm{n}=98)$ & $18(18.4)$ & $29(29.6)$ & $18(18.4)$ & & $13(13.2)$ & $21(21.4)$ & \\
\hline Fever $\geq 38{ }^{\circ} \mathrm{C}(\mathrm{n}=46)$ & $12(26.1)$ & $15(32.6)$ & $11(23.9)$ & & $3(6.5)$ & $4(8.7)$ & $1(2.2)$ \\
\hline Chills $(n=90)$ & $22(24.4)$ & $27(30.0)$ & $20(22.3)$ & & $10(11.1)$ & $8(8.9)$ & $3(3.3)$ \\
\hline Abdominal pain $(n=26)$ & $4(15.4)$ & $4(15.4)$ & 7 (26.9) & & $2(7.7)$ & $7(26.9)$ & $2(7.7)$ \\
\hline Nausea $(\mathrm{n}=33)$ & $7(21.3)$ & $11(33.3)$ & $8(24.2)$ & & $3(9.1)$ & $3(9.1)$ & $1(3.0)$ \\
\hline Insomnia $(\mathrm{n}=8)$ & $3(37.5)$ & $4(50.0)$ & & & & & $1(12.5)$ \\
\hline Sleep disorders $(n=20)$ & $10(50.0)$ & $3(15.0)$ & & & $1(5.0)$ & $5(25.0)$ & $1(5.0)$ \\
\hline \multicolumn{8}{|l|}{ Moderate } \\
\hline Fever $>38^{\circ} \mathrm{C}$ and $<39^{\circ} \mathrm{C}(\mathrm{n}=23)$ & $2(8.7)$ & $8(34.8)$ & $7(30.4)$ & & $5(21.7)$ & $1(4.4)$ & \\
\hline Lymphadenopathy $(\mathrm{n}=23)$ & & $5(21.7)$ & $4(17.4)$ & & $7(30.4)$ & $6(26.1)$ & $1(4.4)$ \\
\hline Myalgia/Arthralgia $(n=115)$ & $17(14.8)$ & $29(25.2)$ & $28(24.3)$ & & $15(13.0)$ & $25(21.7)$ & $1(0.8)$ \\
\hline Local urticaria $(n=2)$ & $1(50.0)$ & $1(50.0)$ & & & & & \\
\hline Vomit $(\mathrm{n}=3)$ & & $2(66.7)$ & & & $1(33.3)$ & & \\
\hline Diarrhoea $(n=21)$ & $6(28.6)$ & $8(38.0)$ & $3(14.3)$ & & $3(14.3)$ & $1(4.8)$ & \\
\hline \multicolumn{8}{|l|}{ Severe } \\
\hline Dyspnoea $(\mathrm{n}=1)$ & & & & & $1(100)$ & & \\
\hline Paraesthesia $(\mathrm{n}=11)$ & $3(27.3)$ & $3(27.3)$ & $2(18.2)$ & & $1(9.0)$ & $2(18.2)$ & \\
\hline Fever $\geq 39^{\circ} \mathrm{C}(\mathrm{n}=4)$ & & $1(25.0)$ & $2(50.0)$ & & $1(25.0)$ & & \\
\hline
\end{tabular}

The higher frequencies among the listed options are reported in bold 
fever $>39{ }^{\circ} \mathrm{C}$ were adverse events reported only after the second dose.

Overall, 247 (72.6\%) participants reported at least one adverse event either after the first, or after the second dose of Comirnaty vaccine. 32 (9.4\%) subjects reported at least one adverse event only after the first dose, while 33 (9.7\%) had adverse events only after the second dose.

Following the second injection, adverse events classified as very common were mostly reported by $80.6 \%$ $(n=274)$ of participants, while common and uncommon events by $22.1 \%(n=75)$ and $2.4 \%(n=8)$, respectively. There was a significant difference for common events reported after the first and second dose $(12.6 \%$ vs $22.1 \%$, $\mathrm{p}=0.0012$ Chi-square test). $53(15.6 \%)$ participants stated additional temporarily coincidental adverse events, not previously described in the literature. The events included cognitive fog $(n=9)$, hot flashes, sweating and breathlessness/shortness of breath at rest or exercise-induced $(n=4)$, widespread itching $(n=3)$, dysgeusia and vertigo $(n=2)$, and sexual/erectile dysfunction, lack of appetite, polydipsia, excessive sweating, hypotension, irritability, malaise, hypertensive peak, transient vision loss/amaurosis fugax, coldness in the lower limbs, haemorrhagic cystitis, severe ovarian pain, tendinitis, ipsilateral axillary pain, pharyngodynia, hyperphagia, chest tightness, rhinitis, sialorrhea, lower limb rash, and anxiety (each $n=1)$.

\section{Adverse Events Reporting, Premedication, and Medication at the Second Dose}

Only $50(17.8 \%)$ individuals who had adverse events after the second dose reported these events to the medical authorities, mainly to occupational physicians $(n=40,80.0 \%)$, followed by family doctors $(n=7,14.0 \%)$, or emergency, Italian Medicine Agency network, and local health authority (each $\mathrm{n}=1,2.0 \%$ ).

Medication before the second vaccine dose was reported by $46(13.5 \%)$ participants, using antihistamines $(n=10$,
Table 4 Correlates of at least one adverse event after the first and second dose of Comirnaty vaccine

\begin{tabular}{|c|c|c|c|c|c|c|}
\hline & \multicolumn{3}{|l|}{ I dose } & \multicolumn{3}{|l|}{ II dose } \\
\hline & $\begin{array}{l}\text { Yes } \\
\text { N }(\%)\end{array}$ & $\begin{array}{l}\text { No } \\
\text { N }(\%)\end{array}$ & $\mathrm{p}$-value & $\begin{array}{l}\text { Yes } \\
\text { N (\%) }\end{array}$ & $\begin{array}{l}\text { No } \\
\text { N }(\%)\end{array}$ & p-value \\
\hline \multicolumn{7}{|l|}{ Sex } \\
\hline Female & $182(87.1)$ & $27(12.9)$ & $0.002 *$ & $181(86.6)$ & $28(13.4)$ & $0.015 *$ \\
\hline Male & $97(74.0)$ & $34(26.0)$ & & $100(76.3)$ & $31(23.7)$ & \\
\hline \multicolumn{7}{|l|}{ Median age } \\
\hline$\leq 49$ years & $153(89.5)$ & $18(10.5)$ & $<0.001 *$ & $150(87.7)$ & $21(12.3)$ & $0.013 *$ \\
\hline$>49$ years & $126(74.6)$ & $43(25.4)$ & & $131(77.5)$ & $38(22.5)$ & \\
\hline \multicolumn{7}{|c|}{ Reference age class } \\
\hline$\leq 55$ years & $196(87.9)$ & $27(12.1)$ & $<0.001 *$ & $197(88.3)$ & $26(11.7)$ & $<0.001 *$ \\
\hline$>55$ years & $83(70.9)$ & $34(31.1)$ & & $84(71.8)$ & $33(28.2)$ & \\
\hline \multicolumn{7}{|c|}{ Professional activity } \\
\hline HCWs & $218(82.9)$ & $45(17.1)$ & $0.461^{*}$ & $223(84.8)$ & $40(15.2)$ & $0.054^{*}$ \\
\hline Non-HCWs & $61(79.2)$ & $16(20.8)$ & & $58(75.3)$ & $19(24.7)$ & \\
\hline \multicolumn{7}{|c|}{ History of SARS-CoV-2 infection } \\
\hline Yes & $18(100)$ & $0(0)$ & $0.052^{\S}$ & $16(88.9)$ & $2(11.1)$ & $0.749^{\S}$ \\
\hline No & $261(81.1)$ & $61(18.9)$ & & $265(82.3)$ & $57(17.7)$ & \\
\hline \multicolumn{7}{|c|}{ Other vaccinations in the past 6 months } \\
\hline Yes & $68(76.4)$ & $21(23.6)$ & $0.106^{*}$ & $66(74.1)$ & $23(25.9)$ & $0.014 *$ \\
\hline No & $211(84.1)$ & $40(15.9)$ & & $215(85.7)$ & $36(14.3)$ & \\
\hline \multicolumn{7}{|c|}{ Any premedication before COVID-19 vaccine } \\
\hline Yes & $23(85.2)$ & $4(14.8)$ & $0.798^{\S}$ & $39(84.8)$ & $7(15.2)$ & $0.681^{\S}$ \\
\hline No & $256(81.8)$ & $57(18.2)$ & & $242(82.3)$ & $52(17.7)$ & \\
\hline \multicolumn{7}{|c|}{ Coexisting acute or chronic disease } \\
\hline Yes & $121(82.3)$ & $26(17.7)$ & $0.915^{*}$ & $118(80.3)$ & $29(19.7)$ & $0.313^{*}$ \\
\hline No & $158(81.9)$ & $35(18.1)$ & & $163(84.5)$ & $30(15.5)$ & \\
\hline \multicolumn{7}{|c|}{ Medical treatment for acute or chronic disease } \\
\hline Yes & $117(81.8)$ & $26(18.2)$ & $0.842^{*}$ & $115(80.4)$ & 28 (19.6) & $0.302 *$ \\
\hline No & $162(82.7)$ & $34(17.3)$ & & $166(84.7)$ & $30(15.3)$ & \\
\hline
\end{tabular}

*Chi-square test; ${ }^{\S}$ Fisher's Exact test; significant results $(\mathrm{p}<0.05)$ are indicated in bold 
Table 5 Factors significantly associated with adverse events classified according to (a) severity and (b) occurrence as reported in the literature following the first and second dose of vaccine

\begin{tabular}{|c|c|c|c|c|c|c|}
\hline \multirow[t]{2}{*}{ (a) } & \multicolumn{3}{|l|}{ I dose } & \multicolumn{3}{|l|}{ II dose } \\
\hline & $\begin{array}{l}\text { Yes } \\
\mathrm{N}(\%)\end{array}$ & $\begin{array}{l}\text { No } \\
\text { N }(\%)\end{array}$ & p-value & $\begin{array}{l}\text { Yes } \\
\mathrm{N}(\%)\end{array}$ & $\begin{array}{l}\text { No } \\
\text { N (\%) }\end{array}$ & $\mathrm{p}$-value \\
\hline \multicolumn{7}{|l|}{ Mild events } \\
\hline \multicolumn{7}{|l|}{ Sex } \\
\hline Female & $179(85.6)$ & $30(14.4)$ & $0.005^{*}$ & $175(83.7)$ & $34(16.3)$ & $0.044 *$ \\
\hline Male & $96(73.3)$ & $35(26.7)$ & & $98(74.8)$ & $33(25.2)$ & \\
\hline \multicolumn{7}{|c|}{ Reference age class } \\
\hline$\leq 55$ years & $193(86.5)$ & $30(13.5)$ & $<0.001 *$ & $193(86.5)$ & $30(13.5)$ & $<0.001 *$ \\
\hline$>55$ years & $82(70.1)$ & $35(29.9)$ & & $80(68.4)$ & 37 (31.6) & \\
\hline \multicolumn{7}{|l|}{ Median age } \\
\hline$\leq 49$ years & $150(87.7)$ & $21(12.3)$ & $0.001 *$ & $146(85.4)$ & $25(14.6)$ & 0.018* \\
\hline$>49$ years & $125(74.0)$ & $44(26.0)$ & & $127(75.1)$ & $42(24.9)$ & \\
\hline \multicolumn{7}{|c|}{ History of SARS-CoV-2 infection } \\
\hline Yes & $18(100)$ & $0(0)$ & $0.030^{\S}$ & $16(88.9)$ & $2(11.1)$ & $0.543^{\S}$ \\
\hline No & $257(79.8)$ & $65(20.2)$ & & $257(79.8)$ & $65(20.2)$ & \\
\hline \multicolumn{7}{|c|}{ Vaccinations in the past 6 months } \\
\hline Yes & $67(75.3)$ & $22(24.7)$ & $0.118^{*}$ & $65(73.0)$ & $24(21.0)$ & $0.045 *$ \\
\hline No & $208(82.9)$ & $43(17.1)$ & & $208(82.9)$ & $43(17.1)$ & \\
\hline \multicolumn{7}{|c|}{ Moderate events } \\
\hline \multicolumn{7}{|c|}{ Sex } \\
\hline Female & $30(14.3)$ & $179(85.7)$ & $0.061 *$ & $97(46.4)$ & $112(53.6)$ & $<0.001 *$ \\
\hline Male & $10(7.6)$ & $121(92.4)$ & & $29(22.1)$ & $102(77.9)$ & \\
\hline \multicolumn{7}{|c|}{ History of SARS-CoV-2 infection } \\
\hline Yes & $9(50.0)$ & $9(50.0)$ & $<0.001^{\S}$ & $10(55.6)$ & $8(44.4)$ & $0.095^{*}$ \\
\hline No & $31(9.6)$ & $291(90.4)$ & & $116(36.0)$ & $206(64.0)$ & \\
\hline \multicolumn{7}{|c|}{ Vaccinations in the past 6 months } \\
\hline Yes & $8(9.0)$ & $81(91.0)$ & $0.344^{*}$ & $25(28.1)$ & $64(71.9)$ & $0.041 *$ \\
\hline No & $32(12.7)$ & $219(87.3)$ & & $101(40.2)$ & $150(59.8)$ & \\
\hline \multicolumn{7}{|l|}{ Severe events } \\
\hline \multicolumn{7}{|l|}{ Sex } \\
\hline Female & $12(5.7)$ & $197(94.3)$ & $0.020 *$ & $15(7.1)$ & $194(92.8)$ & $0.007 *$ \\
\hline Male & $1(0.8)$ & $130(99.2)$ & & $1(0.8)$ & $130(99.2)$ & \\
\hline \multirow[t]{2}{*}{ (b) } & I dose & & & II dose & & \\
\hline & $\begin{array}{l}\text { Yes } \\
\mathrm{N}(\%)\end{array}$ & $\begin{array}{l}\text { No } \\
\text { N (\%) }\end{array}$ & p-value & $\begin{array}{l}\text { Yes } \\
\text { N (\%) }\end{array}$ & $\begin{array}{l}\text { No } \\
\text { N (\%) }\end{array}$ & p-value \\
\hline \multicolumn{7}{|c|}{ Very common events } \\
\hline \multicolumn{7}{|c|}{ Sex } \\
\hline Female & $181(86.6)$ & $28(13.4)$ & $0.004 *$ & $176(84.2)$ & $33(15.8)$ & $\mathbf{0 . 0 3 3} *$ \\
\hline Male & $97(74.0)$ & $34(26.0)$ & & $98(74.8)$ & $33(25.2)$ & \\
\hline \multicolumn{7}{|l|}{ Age } \\
\hline$\leq 49$ years & $152(88.9)$ & $19(11.1)$ & $0.001 *$ & $145(84.8)$ & $26(15.2)$ & $0.049 *$ \\
\hline$>49$ years & $126(74.6)$ & $43(25.4)$ & & $129(76.3)$ & $40(23.7)$ & \\
\hline \multicolumn{7}{|l|}{ Age } \\
\hline$\leq 55$ years & $195(87.4)$ & $28(12.6)$ & $<0.001 *$ & $192(86.1)$ & $31(13.9)$ & $<0.001 *$ \\
\hline$>55$ years & $83(70.9)$ & $34(29.1)$ & & $82(70.1)$ & $35(29.9)$ & \\
\hline \multicolumn{7}{|c|}{ Vaccinations in the past 6 months } \\
\hline Yes & $68(76.4)$ & $21(23.6)$ & $0.127 *$ & $65(73.0)$ & $24(27.0)$ & $0.036 *$ \\
\hline No & $210(83.7)$ & $41(16.3)$ & & $209(83.3)$ & $42(16.7)$ & \\
\hline
\end{tabular}


Table 5 (continued)

\begin{tabular}{|c|c|c|c|c|c|c|}
\hline \multirow[t]{2}{*}{ (b) } & \multicolumn{3}{|l|}{ I dose } & \multicolumn{3}{|l|}{ II dose } \\
\hline & $\begin{array}{l}\text { Yes } \\
\mathrm{N}(\%)\end{array}$ & $\begin{array}{l}\text { No } \\
\text { N }(\%)\end{array}$ & p-value & $\begin{array}{l}\text { Yes } \\
\text { N (\%) }\end{array}$ & $\begin{array}{l}\text { No } \\
\text { N (\%) }\end{array}$ & $\mathrm{p}$-value \\
\hline \multicolumn{7}{|l|}{ Common events } \\
\hline \multicolumn{7}{|l|}{ Sex } \\
\hline Female & $35(16.7)$ & $174(83.3)$ & $0.004 *$ & $59(28.2)$ & $150(71.8)$ & $0.001 *$ \\
\hline Male & $8(6.1)$ & $123(93.9)$ & & $16(12.2)$ & $115(87.8)$ & \\
\hline \multicolumn{7}{|c|}{ Professional activity } \\
\hline HCWs & $36(13.7)$ & $227(86.3)$ & $0.286^{*}$ & $65(24.7)$ & $67(75.3)$ & $0.029 *$ \\
\hline Non-HCWs & $7(9.1)$ & $70(90.9)$ & & $10(13.0)$ & $198(87.0)$ & \\
\hline \multicolumn{7}{|c|}{ History of SARS-CoV-2 infection } \\
\hline Yes & $8(44.5)$ & $10(55.5)$ & $0.001^{\S}$ & $4(28.6)$ & $14(71.4)$ & $>0.999^{\S}$ \\
\hline No & $35(10.9)$ & $287(89.1)$ & & $71(22.0)$ & $251(77.8)$ & \\
\hline \multicolumn{7}{|c|}{ Any COVID-19 symptoms } \\
\hline Yes & $8(53.4)$ & $7(46.6)$ & $<0.001^{\S}$ & $4(26.7)$ & $11(73.3)$ & $0.750^{\S}$ \\
\hline No & $35(10.8)$ & $290(89.2)$ & & $71(21.8)$ & $254(78.2)$ & \\
\hline \multicolumn{7}{|c|}{ Uncommon events } \\
\hline \multicolumn{7}{|l|}{ Sex } \\
\hline Female & $8(3.8)$ & $201(96.2)$ & $0.025^{\S}$ & $6(2.9)$ & $203(97.1)$ & $0.716^{\S}$ \\
\hline Male & $0(0)$ & $131(100)$ & & $2(1.5)$ & $129(98.5)$ & \\
\hline \multicolumn{7}{|c|}{ History of SARS-CoV-2 infection } \\
\hline Yes & $4(22.2)$ & $14(77.8)$ & $<0.001^{\S}$ & $0(0)$ & $18(100)$ & $>0.999^{\S}$ \\
\hline No & $4(1.2)$ & $318(98.8)$ & & $8(2.5)$ & $314(97.5)$ & \\
\hline \multicolumn{7}{|c|}{ Any COVID-19 symptoms } \\
\hline Yes & $4(26.7)$ & $11(73.3)$ & $<0.001^{\S}$ & $0(0)$ & 15 (100) & $>0.999^{\S}$ \\
\hline No & $4(1.2)$ & $321(98.8)$ & & $8(2.4)$ & $317(97.6)$ & \\
\hline
\end{tabular}

${ }^{*}$ Chi-square test; ${ }^{\S}$ Fisher's Exact test; significant results $(\mathrm{p}<0.05)$ are indicated in bold

$21.7 \%)$, cortisone $(n=18,39.1 \%)$, NSAIDs $(n=4,8.7 \%)$, paracetamol $(n=3,6.5 \%)$, antihistamines plus cortisone $(\mathrm{n}=2,4.3 \%)$, antihypertensive plus paracetamol and ranitidine (each $\mathrm{n}=1,2.2 \%$ ). After the second dose administration, $141(50.2 \%)$ participants reporting adverse events $(\mathrm{n}=281)$ undergone a treatment for symptoms, most commonly using paracetamol $(n=101,71.6 \%)$, followed by NSAIDs $(n=21,14.9 \%)$.

\section{Factors Associated with Adverse Events Reported After the First and Second Dose}

Significant differences between adverse events after the first dose and sex were found, as well as with age $\leq 55$ and $\leq 49$ years (Table 4 ). According to the level of severity, mild events correlated with the same variables, with the addition of SARS-CoV-2 history (Table 5a). Occurrence of moderate and severe events correlated with previous SARSCoV-2 infection and sex, respectively (Table 5a).

Concerning the second dose, adverse events correlated with sex, age $\leq 49$ and $\leq 55$ years, and other vaccinations in the previous 6 months (Table 4), and mild events were also associated with these variables (Table 5a). Moderate events were related to sex and with another vaccination in the previous 6 months, while severe events only with sex (Table 5a).

The very common events after either the first or second dose were associated with sex and age $\leq 55$ (Table 5b). These events were related to another vaccination in the previous 6 months only for the second dose. Common events after both doses were associated with sex, and with history of SARSCoV-2 infection and development of any COVID-19 symptom after the first injection, while to professional activity after the second dose (Table 5b). A significant association between occurrence of severe events after the first dose and sex, history of SARS-CoV-2 infection and development of any COVID-19 symptom was observed (Table 5b).

\section{Factors Associated with Specific Adverse Events Reported by Participants After Each Dose}

After the first dose, pain, redness and swelling at the injection site $(p=0.003)$, headache $(p=0.002)$ and chills $(\mathrm{p}=0.007)$ were all associated with sex. Pain, redness and swelling at the injection site were also related to 
age $\leq 49$ years $(\mathrm{p}<0.001)$. Previous COVID-19 symptoms were related to headache $(p=0.016)$, fatigue $(p=0.013)$, and chills $(\mathrm{p}=0.001)$; the latter two events were significantly associated $(p=0.010$ and $p=0.026)$ with vaccinations in the past 6 months and history of SARS-CoV-2 infection. Furthermore, fatigue was related with a medication prior COVID-19 vaccine $(\mathrm{p}=0.008)$. Amongst the moderate events, myalgia/arthralgia correlated with past SARS-CoV-2 infection and COVID-19 symptoms ( $\mathrm{p}<0.001$ for both).

After the second dose, fatigue $(p=0.023)$, headache $(p=0.031)$, chills $(p<0.001)$, abdominal pain $(p=0.012)$, nausea $(\mathrm{p}=0.004)$, fever $>38^{\circ} \mathrm{C}$ and $<39{ }^{\circ} \mathrm{C}(\mathrm{p}=0.031)$, paraesthesia $(\mathrm{p}=0.004)$, and lymphadenopathy and myalgia/ arthralgia ( $\mathrm{p}<0.001$ for both) were significantly associated with sex. Age $\leq 49$ years was related to pain, redness and swelling at the injection site $(\mathrm{p}=0.007)$, fatigue $(\mathrm{p}=0.044)$, fever $<38{ }^{\circ} \mathrm{C}(\mathrm{p}=0.028)$, and lymphadenopathy $(\mathrm{p}=0.055)$. Correlations between age $\leq 55$ years and pain, redness and swelling at the injection site $(\mathrm{p}<0.001)$, fatigue $(\mathrm{p}=0.027)$, fever $<38{ }^{\circ} \mathrm{C}(\mathrm{p}=0.018)$, chills $(\mathrm{p}=0.010)$, and lymphadenopathy $(p=0.025)$ were found. Headache $(p=0.042)$, chills $(p=0.028)$, and myalgia/arthralgia $(p=0.045)$ were associated with past SARS-CoV-2 infection. Interestingly, pain, redness and swelling at the injection site were associated with a coexisting acute/chronic disease $(p=0.016)$, while diarrhoea with any treatment for underlying disease $(\mathrm{p}=0.019)$.

\section{Risk Factors for Specific Adverse Events After Both Injections}

Female sex $(\mathrm{OR}=1.959,95 \%$ CI $1.092-3.516, \mathrm{p}=0.024)$ and age $\leq 55$ years $(\mathrm{OR}=2.942,95 \%$ CI $1.637-5.287$, $\mathrm{p}<0.001)$ significantly increased the likelihood of experiencing at least one adverse event after the first vaccine dose. These factors were also associated with mild events $(\mathrm{OR}=1.856,95 \%$ CI 1.042-3.306, $\mathrm{p}=0.036 ; \mathrm{OR}=3.082$, 95\% CI 1.725-5.506, $\mathrm{p}<0.001$, respectively). Female sex increased the risk of chills $(\mathrm{OR}=3.238,95 \%$ CI 1.33-6.839, $\mathrm{p}=0.002$ ), and of pain, redness and swelling at the injection site $(\mathrm{OR}=1.987,95 \%$ CI $1.170-3.377, \mathrm{p}=0.011)$. For the latter events, age $\leq 49$ years was also a risk factor $(\mathrm{OR}=2.461,95 \%$ CI 1.421-4.261, $\mathrm{p}=0.001)$. Conversely, having received another vaccination in the past 6 months was a protective factor for fatigue $(\mathrm{OR}=0.376,95 \% \mathrm{CI}$ $0.173-0.816, \mathrm{p}=0.013$ ).

After the second dose, age $\leq 55$ years increased the likelihood of at least one and mild adverse events $(\mathrm{OR}=2.818$, 95\% CI 1.587-5.006, $\mathrm{p}<0.001)$. In detail, this age group was a risk factor for pain, redness and swelling at the injection site $(\mathrm{OR}=2.733,95 \%$ CI $1.710-4.367, \mathrm{p}<0.001)$, fever $<38{ }^{\circ} \mathrm{C}(\mathrm{OR}=3.047,95 \%$ CI $1.253-7.310, \mathrm{p}=0.014)$, and chills $(\mathrm{OR}=2.142,95 \%$ CI 1.085-4.227, $\mathrm{p}=0.028)$.
Female sex increased the risk of the occurrence of moderate events $(\mathrm{OR}=2.472,95 \%$ CI 1.456-4.197, $\mathrm{p}=0.001)$, and particularly was associated with myalgia/arthralgia $(\mathrm{OR}=2.270,95 \%$ CI $1.308-3.938, \mathrm{p}=0.004)$. Moreover, age $\leq 49$ years increased the risk of lymphadenopathy $(\mathrm{OR}=3.197,95 \%$ CI 1.127-9.070, $\mathrm{p}=0.029)$.

Risk factors for very common events after the first dose were female sex $(\mathrm{OR}=1.881,95 \%$ CI $1.053-3.359$, $\mathrm{p}=0.033)$ and age $\leq 55$ years $(\mathrm{OR}=2.883,95 \% \mathrm{CI}$ 1.584-5.067, $\mathrm{p}<0.001$ ), with the latter factor also correlating with these events after the second dose $(\mathrm{OR}=2.474$, 95\% CI 1.389-4.406, $\mathrm{p}=0.002$ ). The common reactions after the first and second injection were further associated with female sex $(\mathrm{OR}=3.452,95 \%$ CI $1.395-8.540$, $\mathrm{p}=0.002 ;$ and $\mathrm{OR}=2.145,95 \%$ CI $1.138-4.403, \mathrm{p}=0.018$, respectively).

\section{Discussion}

Various challenges could hamper the objective of immunization of a critical mass of global population, including virus strains escaping the vaccine, limited supply and, above all, the hesitation to be vaccinated because of the likelihood of adverse reactions, partly because none of the COVID19 available vaccines was approved for commercial use in humans before the pandemic. In the placebo-controlled pivotal efficacy trial of Comirnaty vaccine [19], the safety profile monitored over 2 months was similar to other vaccines previously approved, generating short-term and mildto-moderate reactions.

Since the beginning of vaccination campaign in Italy, seven reports of pharmacovigilance on COVID-19 vaccines were published [22], while at time of writing, few observational studies were conducted to identify occurrence and type of adverse events induced by the first and second dose of Comirnaty through an active surveillance that provides the most accurate, complete, and timely information on priority health concerns in a population.

The present study involved employees of private clinics in central Italy to identify number, type, severity, and frequency of adverse events following the immunization with Comirnaty vaccine. As the targeted participants were largely represented by HCWs, the study was also conceived to characterize the reporting of adverse events in a more detailed way due to the high cultural background of this population group. Through active surveillance, nearly $80 \%$ of individuals reported at least one adverse event either after the first or the second dose, in line with previous national reports for the mRNA-based vaccines underlining no substantial differences of rates between both doses [22]. Nevertheless, some reactions were more frequent after the second dose as observed elsewhere [23]. The latest publication from 
the National Medicines Agency indicated that $68 \%$ out of 84,322 adverse reports were referred to Comirnaty, which was the most used vaccine in Italy, with an estimated reporting rate of 137 and 104 every 100,000 administered doses following the first and second dose, respectively [22].

In this survey, participants largely reported very common events as indicated in the literature, especially regarding the first dose, while uncommon events were marginally observed and similarly distributed between both doses. Notably, the common events cited in the literature and in the summary of product characteristic significantly increased after the second dose. The very common events were related to age $\leq 55$ years and female sex after the first dose; in addition, having received another vaccination in the past 6 months was associated with reactions induced by the second dose. The common events reported after the first injection, besides female sex, previous history of SARS-CoV-2 infection, and any COVID-19 symptoms, significantly correlated with being HCWs. This relationship could be associated with the specific group characteristics, including people who are more skilled in health-related concerns, and might also behave differently than the general population.

In this study, vaccine recipients reporting adverse events were largely females with a two-threefold increase in the likelihood to report a reaction as compared to males. These findings were consistent with the latest data of the national pharmacovigilance indicating $72.6 \%$ of the reports of adverse events following Comirnaty vaccination among women compared to $27.4 \%$ in men [22]. The potential sex disparity in vaccine adverse events is not a new occurrence, according to findings related to other vaccines [24, 25], due to a relative suppression of the cellular immune response of males compared to females [26]. These differences may be explained with important biological associations between sex and immune responses [27], and effects on disease susceptibility, transmission, and vaccination outcomes [28]. The major sex hormones seem to exhibit opposing effects on the adaptive and innate immune system, with elevated estradiol increasing and testosterone decreasing the vaccine-induced antibody responses [29, 30]. Moreover, autoimmune disorders are more common reported in women than men [31], together with behavioral attitudes to report adverse events [27]. Females could be also more susceptible to adverse effects due to higher body fat content affecting both volume of distribution and drug clearance [32].

Adverse events were reported amongst $70 \%$ of young adults aged $\leq 55$ years, with a threefold increase as compared to an older population, in agreement with the latest data of national pharmacovigilance reporting that $77 \%$ of adverse reactions were among persons aged 59 years or younger compared to $23 \%$ in those above 60 years [22], and elsewhere [20,33]. Beside a more robust immune system in young individuals [34], there are evidence that old population exhibits a lower ability to establish an effective response to vaccination [35], as demonstrated by a lower frequency of neutralizing antibodies following Comirnaty vaccination compared to younger groups [36].

History of SARS-CoV-2 infection correlated with mild and moderate adverse event after the first dose: all 18 individuals with a past diagnosis with COVID-19 reported mild reactions, and 9 had a moderate event. Nevertheless, this condition was not included in the final multivariate model and should be further investigated comparing convalescent COVID-19 cases and controls. In a study [37], a significant increase of adverse events after vaccination was observed amongst individuals who had previously been infected compared with those who had not, and the number of previously infected subjects who experienced side-effects after the second dose was lower compared with those who were not previously infected. Similar conclusions were observed elsewhere [38] because infected subjects had higher risk of moderate systemic symptoms after the first dose as compared to individuals naive to the virus and showed a lower risk after the second dose. In another observational study [39], prior COVID-19, but not long-COVID, was associated with an increased risk of self-reported reactions, including lymphadenopathy post-vaccination. Additionally, in another prospective study in the UK [20], local post-Comirnaty immunization effects were higher in previously infected individuals than in those without past infection. This correlation could be possibly associated with an increased immunogenicity in individuals with past infection who have developed antibodies with respect to people without past infection [40, 41], as well as with a greater anxiety of side effects, reporting even minimal symptoms.

The study results showed that having received other vaccination in the past 6 months was significantly related to known adverse event following the second dose, but not after the first, particularly mild and moderate reactions. No previous study supported this relationship that should be further investigated since a significant association was not confirmed in the multivariate regression analysis. It has been demonstrated that immunization with some vaccines perturbs the immune system leading to general changes in immune responsiveness [42], and epigenetic modifications may occur in innate immune cells because of vaccination [43].

Concomitant acute/chronic diseases were not associated with adverse events after Comirnaty vaccination, while a significant correlation was only found with pain, redness, and swelling at the injection site after the second dose. Since people with underlying diseases were described as at higher risk of serious outcomes in case of SARS-CoV-2 contagion $[44,45]$, they had priority for receiving COVID-19 vaccine in Italy [46]. Amongst comorbidities, the study participants 
mostly (37\%) were suffering from hypertension, identified as one of the leading diseases in COVID-19 deaths [47].

With respect to the time of onset, the study findings were also in line with national data, reporting that about $80 \%$ of individuals experienced an adverse vaccine event on the same or in the next day of vaccination, and symptoms typically resolved within $48 \mathrm{~h}$ [22], confirming that vaccine-related reactions were not serious. However, as local and systemic transient reactions could occur, this may influence patients' perception of the vaccination, underlining that setting expectations may alleviate some of the potential anxiety-related reactions elicited by post-vaccination reactions [48].

In this survey, most reactions ( $>80 \%$ ) were classified as mild events following both doses with Comirnaty, in line with the latest national report, with $87 \%$ of non-severe reactions, especially among older adults [22]. The national data indicated a rate of $12.8 \%$ of serious adverse events, irrespectively of type of vaccine administered, and particularly 13 cases after Comirnaty vaccine. In the present study, only 13 and 16 cases of serious adverse events following the first and second dose were identified, respectively, which included paresthesia, generalized urticaria, and fever $\geq 39^{\circ} \mathrm{C}$.

Vaccine recipients reported additional temporarily correlated adverse events not previously described in the literature, either after the first or second dose. Cognitive fog, also termed as "vax fog" and referred to brain fog following COVID-19 vaccine, was the most reported reaction. There are no official data yet available on this side effect after Comirnaty vaccine [21], but it may be related to headache-type pain, a more common and previously described side reaction. The study participants further reported face flushing and dizziness. The latter condition could be considered a vague term, encompassing vertigo, disequilibrium, presyncope and light-headedness, which was recently listed among the anxiety-related reactions possibly occurring in the vaccination process itself [21]. According to the Centers for Diseases Control (CDC) [49], $17 \%$ of people who reported side effects indicated that they felt dizzy following the injection, especially after getting the mRNA-based vaccines. Loss of vision (or amaurosis fugax) was further reported among adverse events still not described in the summary of Comirnaty characteristics [21]. No reports on widespread vision-related side effects in association with any COVID-19 vaccines were available, while other vaccines have been linked with rare eyerelated side effects [50]. At least one isolated incident of an eye-related side effect has been reported after Comirnaty vaccine in a $\mathrm{HCW}$ in the United States who experienced eye puffiness after getting vaccine injection [51]. Of note, SARS-CoV-2 infection was reported to cause eye related side effects, as acute vision loss occurred as a secondary consequence to ischemic cerebral infarction among patients after being diagnosed with COVID-19 [52, 53]. Other participants reported sexual or erectile dysfunction, for which no evidence after vaccination were found, while, on the contrary, SARS-CoV-2 infection could pose a risk for both disorders [54, 55], although there is limited research on how the virus could affect the male reproductive system [56, 57]. Hypertensive peak was also reported by the study participants, and alterations in blood pressure were included amongst anxiety-related reactions [21]. There are no immunologic explanations for general vaccinations or mRNA vaccines to cause persistent elevation of blood pressure, but an increase in both diastolic and systolic blood pressure in patients receiving mRNA vaccines was described [58], although blood pressure was not routinely checked before and after the vaccination. Therefore, hypertensive peak could be more likely associated with an increased stress response after administration than with vaccination [59].

In conclusion, findings from this survey were consistent with the current information that short-term adverse events could occur post-immunization with Comirnaty vaccine, and that are moderate in frequency, mild in severity, and short-lived. Adverse reactions were more frequently reported in younger individuals, women, and among those who had prior COVID-19 diagnosis, and generally lasted 1-2 days from the injection. These data could be used to inform people on the likelihood of adverse effects based on their age, sex and first or second dose administration, providing further reassurance on what vaccine recipients might expect.

This study has some limitations. First, the use of selfreported data could possibly introduce misclassification bias, although a good quality of information was gathered due to the level of knowledge and skills related to health issues among the target population, who can better identify post-vaccination reactions. Some participants, such as those who had previously been infected and developed COVID-19 symptoms, might be more likely to report events than others leading to an over-estimation although this constituted only $5 \%$ of the study sample. A precise association between adverse events and past infection could not be estimated due to the limited number of COVID-19 cases. The findings may not completely representative of the general population as included a high proportion of HCWs who could behave differently towards health-related issues, and self-diagnosis. The study design did not allow any inference of causality, also considering that only short-term adverse effects were observed.

Despite this, the survey provides a rapid capture of information during a specific timeframe on adverse events investigated timely closed to vaccination, reducing the likelihood of recall bias. The study remarks that investigating each event after a vaccination provides the opportunity to consolidate the confidence on the safety of COVID-19 approved 
vaccines, as well as to identify any reactions which have not been previously reported.

Acknowledgements The authors would like to thank Dr Jim McLauchlin, Public Health England, Colindale, London, United Kingdom, for the helpful comments and suggestions provided to the manuscript.

Author Contributions GR conceptualized, designed and supervised the study, edited and reviewed the manuscript. MT performed the statistical analysis and interpretation of data, the original draft writing and revised the manuscript. NB assisted data acquisition and curation. CA, VC, FC, MADP, GM, VGM, AN, LR performed data collection and curation, and preliminary analysis. MLS assisted with study design and execution, manuscript revision and supervision. All authors have approved the final article version submitted.

Funding This research did not receive any specific grant from funding agencies in the public, commercial, or not-for-profit sectors.

Data Availability The data that support the findings of this study are available from the corresponding author upon request.

Code Availability Not applicable.

\section{Declarations}

Conflict of interest The authors declare that they have no known competing financial interests or personal relationships that could have appeared to influence the work reported in this paper.

Consent to Participate All participants provided informed consent prior to study participation.

Consent for Publication No individual's data or image are available.

Ethical Approval This study did not require ethics approval as no experimental procedure was applied, and data were anonymously collected from participants who previously signed an informed consent.

\section{References}

1. John Hopkins University \& Medicine. Coronavirus Resource Center. COVID-19 Map. Retrieved August 3, 2021 from https:// coronavirus.jhu.edu/map.html

2. Wilder-Smith, A., Chiew, C. J., \& Lee, V. J. (2020). Can we contain the COVID-19 outbreak with the same measures as for SARS? Lancet Infectious Diseases, 20(5), e102-107. https://doi. org/10.1016/S1473-3099(20)30129-8

3. Kim, K. D., Hwang, I., Ku, K. B., Lee, S., Kim, S. J., \& Kim, C. (2020). Progress and challenges in the development of COVID19 vaccines and current understanding of SARS-CoV-2-specific immune responses. Journal of Microbiology and Biotechnology, 30(8), 1109-1115. https://doi.org/10.4014/jmb.2006.06006

4. Haque, A., \& Pant, A. B. (2020). Efforts at COVID-19 vaccine development: Challenges and successes. Vaccines (Basel), 8(4), 739. https://doi.org/10.3390/vaccines8040739

5. Li, L., Guo, P., Zhang, X., Yu, Z., Zhang, W., \& Sun, H. (2021). SARS-CoV-2 vaccine candidates in rapid development. Human Vaccines \& Immunotherapeutics, 17(3), 644-653. https://doi.org/ $10.1080 / 21645515.2020 .1804777$
6. COVID-19 Vaccine Tracker. Retrieved August 3, 2021 from https://covid19.trackvaccines.org/vaccines/

7. Shimabukuro, T., \& Nair, N. (2021). Allergic reactions including anaphylaxis after receipt of the first dose of Pfizer-BioNTech COVID-19 vaccine. JAMA, 325(8), 780-781. https://doi.org/10. 1001/jama.2021.0600

8. European Medicines Agency. EMA recommends first COVID-19 vaccine for authorisation in the EU. News 21/12/2020. Retrieved July 15, 2021 from https://www.ema.europa.eu/en/news/emarecommends-first-covid-19-vaccine-authorisation-eu

9. Italian Medicines Agency. BioNTech/Pfizer vaccine authorised Answers to frequently asked questions on AIFA's website. Retrieved August 3, 2021 from https://www.aifa.gov.it/en/-/autor izzato-il-vaccino-biontech-pfizer

10. GEDI VISUAL. Retrieved August 4, 2021 from https://lab.gedid igital.it/gedi-visual/2021/covid-19-vaccini-scopri-in-tempo-realequando-saremo-tutti-vaccinati/

11. Radice, A., Carli, G., Macchia, D., \& Farsi, A. (2019). Allergic reactions after vaccination: Translating guidelines into clinical practice. European Annals of Allergy and Clinical Immunology, 51(2), 51-61. https://doi.org/10.23822/EurAnnACI.1764-1489.86

12. Flanagan, K. L., Best, E., Crawford, N. W., et al. (2020). Progress and pitfalls in the quest for effective SARS-CoV-2 (COVID-19) vaccines. Frontiers in Immunology, 11, 579250. https://doi.org/ 10.3389/fimmu.2020.579250

13. Mulligan, M. J., Lyke, K. E., Kitchin, N., et al. (2020). Phase I/II study of COVID-19 RNA vaccine BNT162b1 in adults. Nature, 586, 589-593. https://doi.org/10.1038/s41586-020-2639-4

14. Jackson, L. A., Anderson, E. J., Rouphael, N. G., et al. (2020). An mRNA vaccine against SARS-CoV-2-Preliminary report. New England Journal of Medicine, 383(20), 1920-1931.

15. U.S. Food and Drug Administration. Pfizer-BioNTech COVID-19 Vaccine. Retrieved July 18, 2021 from https://www.fda.gov/emerg ency-preparedness-and-response/coronavirus-disease-2019-covid19/pfizer-biontech-covid-19-vaccine

16. Italian Medicines Agency. Pharmacovigilance on COVID-19 vaccines. Retrieved July 18, 2021 from https://www.aifa.gov.it/en/ farmacovigilanza-vaccini-covid-19

17. Farinazzo, E., Ponis, G., Zelin, E., et al. (2021). Cutaneous adverse reactions after m-RNA COVID-19 vaccine: Early reports from Northeast Italy. European Academy of Dermatology and Venereology, 35(9), e548-e551. https://doi.org/10.1111/jdv.17343

18. Lippi, G., Mattiuzzi, C., \& Henry, B. Mild adverse reactions after COVID-19 vaccination: Updated analysis of Italian Medicines Agency data. Retrieved May 20, 2021 from https://ssrn.com/abstr act $=3817988$ or https: $/ /$ doi.org $/ 10.2139 / \mathrm{ssrn} .3817988$

19. Polack, F. P., Thomas, S. J., Kitchin, N., et al. (2020). Safety and efficacy of the BNT162b2 mRNA Covid-19 vaccine. New England Journal of Medicine, 383, 2603-2615. https://doi.org/10.1056/ NEJMoa2034577

20. Menni, C., Klaser, K., May, A., et al. (2021). Vaccine side-effects and SARS-CoV-2 infection after vaccination in users of the COVID Symptom Study app in the UK: A prospective observational study. Lancet Infectious Diseases, 21(7), 939-949. https:// doi.org/10.1016/S1473-3099(21)00224-3

21. European Medicines Agency. Comirnaty: EPAR—Product information, last updated 20/07/2021. Retrieved August 20, 2021 from https://www.ema.europa.eu/en/medicines/human/EPAR/comir naty

22. Italian Medicines Agency. Pharmacovigilance on COVID-19 vaccines. COVID-19 Vaccine Surveillance Report 7 27/12/2020 26/07/2021. Retrieved August 10, 2021 from https://www.aifa. gov.it/farmacovigilanza-vaccini-covid-19

23. Gee, J., Marquez, P., Su, J., et al. (2021). First month of COVID19 vaccine safety monitoring-United States, December 14 , 
2020-January 13, 2021. Morbidity and Mortality Weekly Report, 70(8), 283-288. https://doi.org/10.15585/mmwr.mm7008e3

24. Halsey, N. A., Griffioen, M., Dreskin, S. C., et al. (2013). Immediate hypersensitivity reactions following monovalent 2009 pandemic influenza A (H1N1) vaccines: Reports to VAERS. Vaccine, 31(51), 6107-6112. https://doi.org/10.1016/j.vaccine.2013.09.066

25. Su, J. R., Moro, P. L., Ng, C. S., Lewis, P. W., Said, M. A., \& Cano, M. V. (2019). Anaphylaxis after vaccination reported to the Vaccine Adverse Event Reporting System, 1990-2016. Journal of Allergy and Clinical Immunology, 143(4), 1465-1473. https:// doi.org/10.1016/j.jaci.2018.12.1003

26. Bouman, A., Heineman, M. J., \& Faas, M. M. (2005). Sex hormones and the immune response in humans. Human Reproduction Update, 11(4), 411-423. https://doi.org/10.3389/fimmu.2018. 02279

27. Cuschieri, S., Borg, M., Agius, S., Souness, J., Brincat, A., \& Grech, V. (2021). Adverse reactions to Pfizer-BioNTech vaccination of healthcare workers at Malta's state hospital. International Journal of Clinical Practice, 75(10), e14605. https://doi.org/10. $1111 / \mathrm{ijcp} .14605$

28. Klein, S. L., \& Flanagan, K. L. (2016). Sex differences in immune responses. Nature Reviews Immunology, 16(10), 626-638. https:// doi.org/10.1038/nri.2016.90

29. Giefing-Kröll, C., Berger, P., Lepperdinger, G., \& Grubeck-Loebenstein, B. (2015). How sex and age affect immune responses, susceptibility to infections, and response to vaccination. Aging Cell, 14(3), 309-321. https://doi.org/10.1111/acel.12326

30. Klein, S. L., Marriott, I., \& Fish, E. N. (2015). Sex-based differences in immune function and responses to vaccination. Transactions of the Royal Society of Tropical Medicine and Hygiene, 109(1), 9-15. https://doi.org/10.1093/trstmh/tru167

31. Angum, F., Khan, T., Kaler, J., Siddiqui, L., \& Hussain, A. (2020). The prevalence of autoimmune disorders in women: A narrative review. Cureus, 12(5), e8094. https://doi.org/10.7759/cureus.8094

32. Zucker, I., \& Prendergast, B. J. (2020). Sex differences in pharmacokinetics predict adverse drug reactions in women. Biology of Sex Differences, 11(1), 32. https://doi.org/10.1186/ s13293-020-00308-5

33. Xiong, X., Yuan, J., Li, M., Jiang, B., \& Lu, Z. K. (2021). Age and gender disparities in adverse events following COVID-19 vaccination: Real-world evidence based on big data for risk management. Frontiers in Medicine (Lausanne), 8, 700014. https://doi.org/10. 3389/fmed.2021.700014

34. Shen-Orr, S. S., \& Furman, D. (2013). Variability in the immune system: Of vaccine responses and immune states. Current Opinion in Immunology, 25(4), 542-547. https://doi.org/10.1016/j.coi. 2013.07.009

35. Gustafson, C. E., Kim, C., Weyand, C. M., \& Goronzy, J. J. (2020). Influence of immune aging on vaccine responses. Journal of Allergy and Clinical Immunology, 145(5), 1309-1321. https:// doi.org/10.1016/j.jaci.2020.03.017

36. Müller, L., Andrée, M., Moskorz, W., et al. (2021). Age-dependent immune response to the Biontech/Pfizer BNT162b2 COVID-19 vaccination. Clinical Infectious Diseases, 2017, ciab381.

37. Ossato, A., Tessari, R., Trabucchi, C., Zuppini, T., Realdon, N., \& Marchesini, F. (2021). Comparison of medium-term adverse reactions induced by the first and second dose of mRNA BNT162b2 (Comirnaty, Pfizer-BioNTech) vaccine: A post-marketing Italian study conducted between 1 January and 28 February 2021. European Journal of Hospital Pharmacy. https://doi.org/10.1136/ejhph arm-2021-002933

38. d'Arminio Monforte, A., Tavelli, A., Perrone, P. M., et al. (2021). Association between previous infection with SARS CoV-2 and the risk of self-reported symptoms after mRNA BNT162b2 vaccination: Data from 3,078 health care workers. EClinicalMedicine, 36, 100914.
39. Raw, R. K., Kelly, C. A., Rees, J., Wroe, C., \& Chadwick, D. R. (2021). Previous COVID-19 infection, but not Long-COVID, is associated with increased adverse events following BNT162b2/ Pfizer vaccination. Journal of Infection. https://doi.org/10.1016/j. jinf.2021.05.035

40. Wise, J. (2021). Covid-19: People who have had infection might only need one dose of mRNA vaccine. BMJ, 372, n308. https:// doi.org/10.1136/bmj.n308

41. Manisty, C., Otter, D., Treibel, T. A., et al. (2021). Antibody response to first BNT162b2 dose in previously SARS-CoV-2-infected individuals. Lancet, 397(10279), 1057-1058. https://doi. org/10.1016/S0140-6736(21)00501-8

42. Pollard, A. J., Finn, A., \& Curtis, N. (2017). Non-specific effects of vaccines: Plausible and potentially important, but implications uncertain. Archives of Disease in Childhood, 102(11), 1077-1081. https://doi.org/10.1136/archdischild-2015-310282

43. Pollard, A. J., \& Bijker, E. M. (2021). A guide to vaccinology: From basic principles to new developments. Nature Reviews Immunology, 21(2), 83-100. https://doi.org/10.1038/ s41577-020-00479-7

44. Sun, K., Chen, J., \& Viboud, C. (2020). Early epidemiological analysis of the coronavirus disease 2019 outbreak based on crowdsourced data: A population-level observational study. Lancet Digital Health, 2(4), e201-208. https://doi.org/10.1016/S25897500(20)30026-1

45. Emami, A., Javanmardi, F., Pirbonyeh, N., \& Akbari, A. (2020). Prevalence of underlying diseases in hospitalized patients with COVID-19: a systematic review and meta-analysis. Archives of Academic Emergency Medicine, 8(1), e35.

46. Italian Medicines Agency. Vaccination with Pfizer (Comirnaty) COVID-19 mRNA BNT162b2 vaccine AIFA FAQs. Retrieved August 20, 2021 from https://www.aifa.gov.it

47. Epicentro, Istituto Superiore di Sanità. Caratteristiche dei pazienti deceduti positivi all'infezione da SARS-CoV-2 in Italia, updated on 21 July 2021. Retrieved August 20, 2021 from https://www. epicentro.iss.it/coronavirus/sars-cov-2-decessi-italia\#4

48. Chapin-Bardales, J., Gee, J., \& Myers, T. (2021). Reactogenicity following receipt of mRNA-based COVID-19 vaccines. JAMA, 32(21), 2201-2202. https://doi.org/10.1001/jama.2021.5374

49. Centers for Diseases Control and Prevention. Interim clinical considerations for use of COVID-19 vaccines currently authorized in the United States. Retrieved August 19, 2021 from https://www. cdc.gov/vaccines/covid-19/clinical-considerations/covid-19-vacci nes-us.html

50. Looking Glass Optical Eye Care Center, updated on 26 April 2021. Retrieved August 19, 2021 from https://www.lookinggla ssoptical.com/covid-19-vaccine-affect-your-vision/

51. Abc news. Health officials track safety as COVID-19 vaccines roll out. Retrieved August 20, 2021 from https://abcnews.go.com/ $\mathrm{amp} /$ Health/wireStory/health-officials-track-safety-covid-19-vacci nes-roll-74767373

52. Selvaraj, V., Sacchetti, D., Finn, A., \& Dapaah-Afriyie, K. (2020). Acute Vision Loss in a Patient with COVID-19. Rhode Island Medical Journal (2013), 103(6), 37-38.

53. Cyr, D. G., Vicidomini, C. M., Siu, N. Y., \& Elmann, S. E. (2020). Severe bilateral vision loss in 2 patients with coronavirus disease 2019. Journal of Neuro-Ophthalmology, 40(3), 403-405.

54. The conversation. COVID-19 could cause male infertility and sexual dysfunction-But vaccines do not, updated on 26 July 2021. Retrieved August 20, 2021 from https://theconversation. $\mathrm{com} /$ covid-19-could-cause-male-infertility-and-sexual-dysfu nction-but-vaccines-do-not-164139

55. Factcheck.org. Research rebuts claims linking COVID-19 vaccines to male infertility, updated on 17 June 2021. Retrieved August 20, 2021 from https://www.factcheck.org/2021/06/scicheck-resea 
rch-rebuts-baseless-claims-linking-covid-19-vaccines-to-maleinfertility/

56. Kresch, E., Achua, J., Saltzman, R., et al. (2021). COVID-19 endothelial dysfunction can cause erectile dysfunction: Histopathological, immunohistochemical, and ultrastructural study of the human penis. World Journal of Men's Health, 39(3), 466-469. https://doi.org/10.5534/wjmh.210055

57. Achua, J. K., Chu, K. Y., Ibrahim, E., et al. (2021). Histopathology and ultrastructural findings of fatal COVID-19 infections on testis. World Journal of Men's Health, 39(1), 65-74. https://doi.org/10. 5534/wjmh. 200170

58. Meylan, S., Livio, F., Foerster, M., et al. (2021). Stage III hypertension in patients after mRNA-based sars-cov-2 vaccination.
Hypertension, 77(6), e56-57. https://doi.org/10.1161/HYPER TENSIONAHA.121.17316

59. American Academy of Allergy Asthma \& Immunology. Hypertension after mRNA COVID-19 vaccine. Retrieved August 20, 2021 from https://www.aaaai.org/allergist-resources/ask-the-expert/ answers/2021/hypertension

Publisher's Note Springer Nature remains neutral with regard to jurisdictional claims in published maps and institutional affiliations. 